\title{
Cultural Malpractices During Pregnancy, Child Birth and Postnatal Period Among Women of Child Bearing Age in Limmu Genet Town, Southwest Ethiopia
}

\author{
Tadesse Nigussie Tola, Andualem Henok Tadesse* \\ Department of Public health, Mizan-Tepi University, Mizan, Ethiopia
}

Email address:

andualemhenok@gmail.com (A. Henok)

To cite this article:

Tadesse Nigussie Tola, Andualem Henok Tadesse. Cultural Malpractices During Pregnancy, Child Birth and Postnatal Period Among Women of Child Bearing Age in Limmu Genet Town, Southwest Ethiopia. Science Journal of Public Health. Vol. 3, No. 5, 2015 , pp. $752-756$. doi: $10.11648 /$ j.sjph.20150305.32

\begin{abstract}
Back ground: Everyday, at least 800 women die worldwide from the complications of pregnancy \& child birth, $90 \%$ of which occurring in Asia \& Sub Saharan Africa. These shows, maternal death in developing country is high. One of the contributing factors for these problems is cultural malpractices during pregnancy and child birth. The actual incidence of cultural malpractices in developing countries accounts at about 5-15\% of maternal deaths. Objective: To assess prevalence and factors associated with cultural malpractice practiced during pregnancy, child birth and postnatal period among women of child bearing age in Limmu Genet town, Southwest Ethiopia. Methods: Community based cross sectional study was conducted to determine prevalence and factors associated with cultural malpractices that take place during pregnancy, child birth and postnatal period among women of the reproductive age group. The study was conducted from June to September 2014. Data was collected by using interviewer administered pretested questionnaire by trained high school students. The collected data was entered to Epidata 3.1 and transported to SPSS version 17 for data analysis. Data was presented by using tables and graphs. The association between variables was tested by using $\mathrm{X}^{2}$ test with a p-value of less than 0.05 was used to declare the significance of the association. Result: Out of 303 women 58(19.1\%) practiced nutritional taboo, 67(22\%) women practiced abdominal massage and 116(38.3\%) delivered their babies at home, 33(28.4\%) washed their babies immediately after birth and 26(22.41\%) did not give collostrum to new born. Educational status was significantly associated with nutritional taboo, abdominal massage, home delivery and avoiding colostrum feeding to new born. Conclusion: The prevalence of cultural malpractices during pregnancy, delivery and postpartum in the study area was high. Therefore health education and promoting formal female education are important to decrease or avoid these cultural malpractices.
\end{abstract}

Keywords: Cultural Malpractice, Pregnancy, Labour, Postpartum, Ethiopia

\section{Introduction}

Globally, over 300,000 maternal deaths occur each year. This figure translates in to more than 1400 child birth deaths every 24 hours. Nearly half of all maternal deaths occur in Africa. For every woman who dies, another 100 women survive childbearing but suffer from serious disease, disability, or physical damage caused by pregnancy related complications. Maternal morbidity in the form of uterine prolapse, pelvic inflammatory diseases, vesico vaginal fistula, urinary and fecal incontinence, infertility and pain during intercourse are few of the long term consequences of pregnancy related complication. A million or more children are left motherless each year when mother dies from pregnancy related causes. Thus the United Nations' millennium developmental goals (MDGs) set in 2000 targeted a $75 \%$ reduction in the maternal mortality ratio by 2015 (1,2). Every day at least 800 women are dying worldwide from the complication of pregnancy and child birth, of which majority occurring in Asia and Sub Saharan Africa. Maternal mortality rate shows the largest between developed and developing countries. In Ethiopia, maternal and infant mortality and morbidity level are the highest in the world. The maternal mortality ratio in the year 2005 was 673 per 100,000 live births and IMR 59 deaths per 1000 live births $(1,3)$. 
Many mothers suffer from infection of the reproductive tract and neonatal sepsis due to unclean environment and inappropriate care during pregnancy and delivery, which, infant improperly delivered by unskilled birth attendants, and/or the traditional practices performed during delivery are the major cause of sepsis and death. The actual incidence in developing country is not known, but it accounts $5-15 \%$ (3). Some of the determinant factor for this are the general socio -economic conditions, educational status, the believes of the community on modern health care service \& enabling to use the available modern health care service, the socio cultural believes of the community and simply using cultural way of treating the mother during pregnancy, delivery and postpartum period. In the same way such cultural practices may be accompanied by numerous complications which result to permanent damage to the body and even death. On the other hand, pregnancy by itself has not been considered as a risk for maternal health in Africa during pregnancy (4). A life time risk for maternal death in developed countries is forty times higher than that of developed countries. Bleeding, obstructed labor, hypertensive disorder ( pregnancy toxemia), unsafe abortion, sepsis and home delivery contribute for up to $80 \%$ of maternal death with resultant increased fetal loss, prenatal mortality and poor survival of children (5). Cultural practices during pregnancy and child birth are the common practices in the developing countries. This cultural effect may be beneficial, neutral, or harmful. Despite the lack of precise information, harmful cultural practices have a serious effect on the health of pregnant women, commonly in developing country like Ethiopia. As in developing countries, in Ethiopia, the great majority of women deliver at home and follow the cultural birth customs. This create problems for several years that we couldn't overcome much of the risk which is associated with harmful cultural practices which are performed at each individual's home during delivery. Thus the benefits of modern maternity care have been influenced largely by the cultural practices under

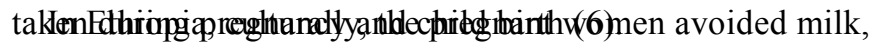
honey, meat and some valuable food stuffs in order to avoid large baby and difficulty of labor. Her abdomen is massaged to relieve back pain and to correct the position of the fetus mostly by untrained traditional birth attendants. In addition to this, different herbal medicines or drugs are taken during pregnancy and child birth in many regions as treatment for some pregnancy and pregnancy related symptoms. Also the pregnant women do extraneous working activity till the last trimester of pregnancy because they believe that it will help to make the labor easier and reduce the body weight of the infant (7). In Ethiopia, data on cultural practice during pregnancy and child birth is not completely understood (in complete) and little is known about either beneficial or full aspect both in local and national level. No study was conducted in Limmu Genet and around it to assess the problem. Thus this study will attempt and help to determine the type and magnitude of cultural malpractice performed during pregnancy, child birth and immediate post natal time among reproductive age group women of an area. As it is seen in other part of Ethiopia, in Limmu Genet and around there may be different cultural practices which is performed during pregnancy and /or child birth, but there is no data which clearly indicate the types \& prevalence of cultural malpractice and their influencing factors and put base line for further research in order to recommend the necessary intervention to concerned body. In Ethiopia, different cultural practices have been under taken during pregnancy child birth in almost all the regions. But there is no study done here which clearly explain it. Therefore purpose of study was to assess the prevalence and factors associated with cultural malpractices during pregnancy, birth and postpartum in study area. It is hoped that the result of this study will provide program and policy makers with data on cultural malpractices.

\section{Methods and Materials}

\subsection{Study Area and Period}

The study was conducted in Limmu Genet town, Jimma zone, Oromia region, Southwest Ethiopia. The town has two kebeles and total population of 11,991. There were 1304 women of reproductive age. It is $75 \mathrm{~km}$ from Jimma town and $435 \mathrm{~km}$ from Addis Ababa. The town has one district hospital, one health center and five private clinics. The study was conducted from June to September 2014.

\subsection{Study Design}

The study design was community based cross-sectional.

\subsection{Source Population}

Source population was all women of reproductive age group in Limmu Genet town who experienced at least one pregnancy.

\subsection{Study Population}

All randomly selected women of reproductive age (15-49) who experienced at least one pregnancy in Limmu Genet town.

\subsection{Sample Size and Sampling Technique}

Sample size was calculated by using single population proportion formula to calculate sample size.

$$
\begin{aligned}
& \mathrm{n}=\frac{z^{2} p q}{d^{2}}=384 \\
& \mathrm{nf}=\frac{n \times N}{N \pm n}=\frac{384 \times 1304}{384 \pm 1304}=296 \\
& \mathrm{nf}=296+14.8=312 \\
& \mathrm{n}=\text { required sample size } \\
& \mathrm{p}=\text { proportion }=0.5 \\
& \mathrm{~d}=\text { marginal error }=0.05 \\
& \mathrm{z}=\text { standard score }=1.96 \\
& \mathrm{~N}=\text { finite population size }(1304)
\end{aligned}
$$


To select 312 women systematic random sampling was conducted by considering list of women with at least one pregnancy as sampling frame.

\subsection{Data Collection}

Data was collected using structured questionnaires prepared in English and translated to Afan Oromo (local language). The questionnaire was pretested before commencement of actual data collection. The questionnaire contains three parts: socio demographic characteristics, reproductive health part and practices of cultural malpractices. Data collection was conducted after obtaining permission from concerned officials. Five data collectors from high school students trained about the study and on data collection.

\subsection{Data Quality Control}

One week before actual survey pretest was carried out in $5 \%$ of study population and necessary modification was made before going to apply on the study subject. Data was collected carefully by the principal investigators and trained high school students to get the reliable and necessary in formation according to the aim of the study. The principal investigator had made an ongoing checking each day during the data collection to ensure the quality of data by checking filled questionnaires.

\subsection{Data Analysis}

The collected data was entered to Epidata 3.1 software and then transported to SPSS version 17 for data analysis. Frequencies were calculated to determine prevalences. Association between variables was tested by using $\mathrm{x}^{2}$ test at $95 \%$ confidence level and a p-value of less than 0.05 was used to declare the significance of the association. Finally tables were used to present the findings.

\subsection{Ethical Consideration}

Ethical clearance and formal letter were obtained from Jimma University. Letter was also obtained from the administrative counsel of Limmu Genet administration. Confidentiality of the respondents was assured that any person's name was not appeared on research documents and respondents was informed about the aim of the study.

\section{Results}

\subsection{Socio Demographic Characteristics}

Regarding to the socio demographic characteristics of the respondents, $107(35.3 \%)$ were in the age group of 25- 29 . Majority of them were Muslim 119(39.3\%) and Oromo $177(58.4 \%)$ by religion and ethinicity respectively. Out of 303 , 265(87.5\%) were married and $157(51.8 \%)$ completed grade 1 - 8 . Concerning occupation $187(58.7 \%)$ were house wives (Table 1).

\subsection{Cultural Malpractices During Pregnancy}

This study assessed the respondents' experiences of malpractices during pregnancy. Out of 303 women 58 (19.1\%) practiced nutritional taboo, 67(22\%) women practiced abdominal massage and $116(38.3 \%)$ delivered their babies at home (Table 2).

Table 1. Socio demographic characteristics of respondents in Limmu Genet town, 2014.

\begin{tabular}{|c|c|c|c|}
\hline Characteristics & & Number & Percent \\
\hline \multirow{6}{*}{ Age } & $15-19$ & 4 & 1.3 \\
\hline & $20-24$ & 92 & 31 \\
\hline & $25-29$ & 105 & 35.3 \\
\hline & $30-34$ & 48 & 16.2 \\
\hline & $35-39$ & 51 & 16.8 \\
\hline & $40-44$ & 8 & 2.4 \\
\hline \multirow{4}{*}{ Religion } & Muslim & 119 & 39.3 \\
\hline & Orthodox & 112 & 37 \\
\hline & Protestant & 49 & 16.2 \\
\hline & Others & 23 & 7.5 \\
\hline \multirow{4}{*}{ Ethnicity } & Oromo & 177 & 58.4 \\
\hline & Amhara & 63 & 20.8 \\
\hline & Gurage & 35 & 11.6 \\
\hline & Others & 23 & 8.2 \\
\hline \multirow{4}{*}{ Educational status } & Illiterate & 111 & 36.6 \\
\hline & G $1-8$ & 157 & 51.8 \\
\hline & G 9-12 & 21 & 6.9 \\
\hline & $12+$ & 14 & 4.7 \\
\hline \multirow{4}{*}{ Marital status } & Single & 9 & 2.8 \\
\hline & Married & 265 & 87.5 \\
\hline & Divorced & 14 & 4.6 \\
\hline & Widowed & 15 & 4.9 \\
\hline \multirow{5}{*}{ Occupational status } & House wife & 187 & 58.7 \\
\hline & Student & 12 & 4 \\
\hline & Employer & 39 & 12.9 \\
\hline & Farmer & 46 & 15.2 \\
\hline & Merchant & 28 & 9.2 \\
\hline
\end{tabular}

Table 2. Prevalence of cultural malpractices during pregnancy and child birth among women of Limmu town, 2014.

\begin{tabular}{lllll}
\hline \multirow{2}{*}{$\begin{array}{l}\text { Cultural malpractices } \\
\text { during pregnancy }\end{array}$} & \multicolumn{3}{l}{ Responses } \\
\cline { 2 - 5 } & Yes & No & \\
\cline { 2 - 5 } & Number & \% & Number & \% \\
\hline Food taboos & 58 & 19.1 & 245 & 88.9 \\
$\begin{array}{l}\text { Abdominal massage } \\
\text { Home delivery }\end{array}$ & 67 & 22 & 236 & 78 \\
$\begin{array}{l}\text { Cutting cord by unclean } \\
\text { blade }(\mathrm{N}=116)\end{array}$ & 116 & 38.3 & 187 & 61.7 \\
$\begin{array}{l}\text { Avoiding } \\
\text { collestrum(N=116) }\end{array}$ & 5 & 4.31 & 111 & 95.69 \\
$\begin{array}{l}\text { Washing baby immediately } \\
\text { after delivery(N=116) }\end{array}$ & 26 & 22.41 & 90 & 77.58 \\
\hline
\end{tabular}

\subsection{Cultural Malpractices During Delivery and Post Natal Period}

From 116 women who gave birth at home 33(28.44\%) washed their babies immediately after birth. About $26(22.41 \%)$ did not give collustrum to new born and $5(4.31 \%)$ cut umbilical cord by unsterile material (Table 2). 


\subsection{Factors Associated with Cultural Malpractics}

Nutritional taboo was cross tabulated against sociodemographic characteristics. The $\mathrm{x}^{2}$ test was conducted to assess if there was significant association between them. There was significant association between age and educational status of respondents and nutritional taboo (Table 3). Age group and educational status were significantly associated with home delivery and abdominal massage (Table 4 and 5). Age group, educational status and ethnicity were significantly associated with prohibiting colostrum(Table 6).

Table 3. Association of nutritional taboo with age group, religion, Educational status and ethnicity among Limmu genet women, 2014.

\begin{tabular}{|c|c|c|c|c|c|}
\hline \multirow{2}{*}{ Characters } & & \multicolumn{2}{|c|}{ Nutritional taboos } & \multirow{2}{*}{ Total } & \multirow{2}{*}{ P value } \\
\hline & & Yes & No & & \\
\hline \multirow{7}{*}{ Age } & $15-19$ & 0 & 4 & 4 & \multirow{7}{*}{$<0.05$} \\
\hline & $20-24$ & 6 & 86 & 92 & \\
\hline & $25-29$ & 21 & 84 & 105 & \\
\hline & $30-34$ & 19 & 29 & 48 & \\
\hline & $35-39$ & 10 & 41 & 51 & \\
\hline & $40-44$ & 2 & 6 & 8 & \\
\hline & Total & 58 & 245 & 303 & \\
\hline \multirow{5}{*}{ Religion } & Muslim & 29 & 90 & 119 & \multirow{5}{*}{$>0.05$} \\
\hline & Orthodox & 17 & 75 & 112 & \\
\hline & Protestant & 5 & 44 & 49 & \\
\hline & Others & 3 & 20 & 23 & \\
\hline & Total & 58 & & & \\
\hline \multirow{2}{*}{$\begin{array}{l}\text { Educatiuonal } \\
\text { status }\end{array}$} & Illiterate & 49 & 62 & 111 & \multirow[b]{2}{*}{$<0.05$} \\
\hline & $\begin{array}{l}\text { Primary } \\
\text { school }\end{array}$ & 9 & 148 & 157 & \\
\hline \multirow{4}{*}{ Ethinicity } & Oromo & 27 & 150 & 177 & \multirow{4}{*}{$>0.05$} \\
\hline & Amhara & 21 & 42 & 63 & \\
\hline & Gurage & 4 & 31 & 35 & \\
\hline & Others & 6 & 21 & 23 & \\
\hline
\end{tabular}

Table 4. Association of Abdominal Massage with age group, religion, Educational status and ethnicity among Limmu Genet women June 2013 G.C.

\begin{tabular}{|c|c|c|c|c|c|}
\hline \multirow[t]{2}{*}{ Characters } & & \multicolumn{2}{|c|}{$\begin{array}{l}\text { Abdominal } \\
\text { massage }\end{array}$} & \multirow[t]{2}{*}{ total } & \multirow[t]{2}{*}{ P value } \\
\hline & & Yes & No & & \\
\hline \multirow{6}{*}{ Age } & $15-19$ & 0 & 4 & 4 & \multirow{6}{*}{$<0.05$} \\
\hline & $20-24$ & 3 & 89 & 92 & \\
\hline & $25-29$ & 14 & 91 & 105 & \\
\hline & $30-34$ & 16 & 32 & 48 & \\
\hline & $35-39$ & 27 & 24 & 51 & \\
\hline & $40-44$ & 7 & 1 & 8 & \\
\hline \multirow{4}{*}{ Religion } & Muslim & 26 & 93 & 119 & \multirow{4}{*}{$>0.05$} \\
\hline & Orthodox & 24 & 88 & 112 & \\
\hline & Protestant & 12 & 37 & 49 & \\
\hline & Others & 5 & 18 & 23 & \\
\hline \multirow{2}{*}{$\begin{array}{l}\text { Educational } \\
\text { status }\end{array}$} & Illiterate & 51 & 60 & 111 & \multirow{2}{*}{$<0.05$} \\
\hline & $1-8$ & 16 & 141 & 157 & \\
\hline \multirow{4}{*}{ Ethnicity } & Oromo & 25 & 152 & 177 & \multirow{4}{*}{$>0.05$} \\
\hline & Amhara & 21 & 42 & 63 & \\
\hline & Gurage & 14 & 21 & 35 & \\
\hline & Others & 9 & 18 & 23 & \\
\hline
\end{tabular}

Table 5. Association of home delivery with age group, religion, educational status and ethnicity among Limmu Genet women, 2014.

\begin{tabular}{|c|c|c|c|c|c|c|}
\hline \multirow[t]{2}{*}{ № } & \multirow[t]{2}{*}{ Characters } & & \multicolumn{2}{|c|}{$\begin{array}{l}\text { Home } \\
\text { delivery }\end{array}$} & \multirow[t]{2}{*}{ total } & \multirow[t]{2}{*}{ P value } \\
\hline & & & Yes & No & & \\
\hline \multirow{6}{*}{1} & \multirow{6}{*}{ Age } & $15-19$ & 0 & 4 & 4 & \multirow{6}{*}{$<0.05$} \\
\hline & & $20-24$ & 6 & 86 & 92 & \\
\hline & & $25-29$ & 46 & 59 & 105 & \\
\hline & & $30-34$ & 23 & 25 & 48 & \\
\hline & & $35-39$ & 35 & 16 & 51 & \\
\hline & & $40-44$ & 6 & 2 & 8 & \\
\hline \multirow{4}{*}{2} & \multirow{4}{*}{ Religion } & Muslim & 45 & 74 & 119 & \multirow{4}{*}{$>0.05$} \\
\hline & & Orthodox & 42 & 70 & 112 & \\
\hline & & Protestant & 18 & 31 & 49 & \\
\hline & & Others & 11 & 12 & 23 & \\
\hline \multirow{2}{*}{3} & \multirow{2}{*}{ Educational } & Illiterate & 83 & 28 & 111 & \multirow{2}{*}{$<0.05$} \\
\hline & & $1-8$ & 33 & 124 & 157 & \\
\hline \multirow{4}{*}{4} & \multirow{4}{*}{ Ethnicity } & Oromo & 68 & 109 & 177 & \multirow{4}{*}{$>0.05$} \\
\hline & & Amhara & 29 & 34 & 63 & \\
\hline & & Gurage & 9 & 26 & 35 & \\
\hline & & Others & 5 & 22 & 23 & \\
\hline
\end{tabular}

Table 6. Association of avoiding collestrum from new born with age group, religion, educational status and ethnicity among Limmu Genet women, 2014.

\begin{tabular}{|c|c|c|c|c|c|}
\hline \multicolumn{2}{|l|}{ Characters } & \multicolumn{2}{|c|}{$\begin{array}{l}\text { Giving collestrum } \\
\text { for new born }\end{array}$} & \multirow[t]{2}{*}{ Total } & \multirow[t]{2}{*}{$P$ value } \\
\hline \multirow{7}{*}{ Age } & & Yes & no & & \\
\hline & $15-19$ & 4 & 0 & 4 & \multirow{6}{*}{$<0.05$} \\
\hline & $20-24$ & 90 & 2 & 92 & \\
\hline & $25-29$ & 101 & 4 & 105 & \\
\hline & $30-34$ & 35 & 13 & 48 & \\
\hline & $34-39$ & 47 & 4 & 51 & \\
\hline & $40-44$ & 5 & 3 & 8 & \\
\hline \multirow{4}{*}{ Religion } & Muslim & 107 & 12 & 119 & \multirow{4}{*}{$>0.05$} \\
\hline & Orthodox & 105 & 7 & 112 & \\
\hline & Protestant & 45 & 4 & 49 & \\
\hline & Others & 20 & 3 & 23 & \\
\hline \multirow{2}{*}{$\begin{array}{l}\text { Educational } \\
\text { status }\end{array}$} & Illiterate & 93 & 18 & 111 & \multirow{2}{*}{$<0.05$} \\
\hline & $1-8$ & 149 & 8 & 157 & \\
\hline \multirow{4}{*}{ Ethnicity } & Oromo & 166 & 11 & 177 & \multirow{4}{*}{$<0.05$} \\
\hline & Amhara & 54 & 9 & 63 & \\
\hline & Gurage & 31 & 4 & 35 & \\
\hline & Others & 25 & 2 & 23 & \\
\hline
\end{tabular}

\section{Discussion}

This community based study has attempted to assess the prevalence and associated factors of cultural malpractices during pregnancy, delivery and postnatal period in Limmu Genet town, Jimma Zone, Southwest Ethiopia.

The result of the study revealed that the prevalence of nutritional taboo was $19.1 \%$. This was lower than the study conducted in Shashemene which was $49.8 \%$ (8). But even if it seems lower, nutritional taboos have rampant effect on the outcome pregnancy. In our study nutritional taboo was significantly associated with educational status of mothers. This goes in line with the study conducted in Shashemene (8). This indicates that education has impact on avoiding nutritional taboos during pregnancy and hence avoid iits 
consequences.

Our study indicated that the prevalence of home delivery was $38.3 \%$. This was lower than the study conducted in Arbaminch zuria in south Ethiopia (9). In our study educational status of mothers was significantly associated with home delivey. This result was similar with the study conducted in Arbaminch, South Ethiopia.

This study revealed that the prevalence of abdominal massage during pregnancy was $22 \%$. This was higher than the study conducted in Nigeria which was $14.79 \%$ (10). This indicates that our study participants were practicing abdominal massage apparently. This may cause adverse outcomes during pregnancy.

In the study conducted in Bangeladesh the prevalence of not giving colostrum was $41 \%$ (11). This was higher than the result of our study which was $22.41 \%$. But our study result was higher than the study conducted in North Ethiopia on complementary feeding which was $10.75 \%$ (12).

In this study $28.4 \%$ washed their babies immediately after birth. This may cause negative effect on the new born. About $4.3 \%$ of respondents used unsterile material to cut the umblical cord during home delivery.

Overall this study showed that educational status was significantly associated with nutritional taboo, abdominal massage, home delivery and avoiding colostrum feeding to new born. This indicates that empowerment of women interms of education has unreplacable role in preventing cultural malpractices during pregnancy, delivery and postpartum. This is because poor knowledge is associated with cultural malpractices (13).

\section{Conclusion}

The prevalence of cultural malpractices during pregnancy, delivery and postpartum in the study area was high. Educational status was significantly associated with nutritional taboo, abdominal massage, home delivery and avoiding colotrum feeding to new born. Therefore health education and promoting formal female education are important to decrease or avoid these cultural malpractices.

\section{References}

[1] www.unfpa.org/public/home/mothers/MMEstimates2012
[2] Central statistical authority (CSA) \& Ethiopian demographic $\&$ health survey 2005 Addis Abeba, Ethiopia.

[3] Central statistical authority (CSA) \& Ethiopian demographic $\&$ health survey 2011 Addis Abeba, Ethiopia.

[4] Ethiopian public health training initiative (EPHTI), Harm full traditional practice, manual on HTP for health center team, Awasa College 2005.

[5] www.ncbi.nlm.nih.gov/pubmed/8693724

[6] Keno D., cultural practices during pregnancy \& child birth among WCBA in shebe town, 1998, research report summated to department of health officer as practical fulfillment of BSC in public health ( un published)

[7] Zein A, Helmat k, Ecology of health and disease in Ethiopia 1998

[8] Nejimu Biza Zepro. Food Taboos and Misconceptions Among Pregnant Women of Shashemene District, Ethiopia, 2012. Science Journal of Public Health. Vol. 3, No. 3, 2015, pp. 410-416. doi: 10.11648/j.sjph.20150303.27

[9] Gistane Ayele, Maralign Tilahune, Behailu Merdikyos, Worku Animaw, Wondimagegn Taye. Prevalence and Associated Factors of HomeDelivery in Arbaminch Zuria District, Southern Ethiopia: Community Based Cross Sectional Study. Science Journal of Public Health. Vol. 3, No. 1, 2015, pp. 6-9. doi: 10.11648/j.sjph.20150301.12

[10] Ugboma HA, Akani CI. Abdominal massage: another cause of maternal mortality. Niger J Med;13(3):259-62. 2004

[11] Holman DJ, Grimes MA. Colostrum feeding behaviour and initiation of breast-feeding in rural Bangladesh.. J Biosoc Sci.;33(1):139-54 2001.

[12] Ergib Mekbib, Ashenafi Shumey, Semaw Ferede, Fisaha Haile. Magnitude and Factors Associated with Appropriate ComplementaryFeeding among Mothers Having Children 6-23 Months-of-Age in Northern Ethiopia; A Community-Based Cross-Sectional Study.Journal of Food and Nutrition Sciences. Vol. 2, No. 2, 2014, pp. 36-42. doi: 10.11648/j.jfns.20140202.13

[13] Nurilign Abebe Moges, Getachew Mullu, Mihiretie Gedfew, Mohammednur Redi, Mohammed Molla, Setarg Ayenew, Shegaw Fentahun, Solomon Adisie, Zewudu Dagnew. Knowledge, Attitude and Practice of Women Towards Female Genital Mutilation in Lejet Kebele, Dembecha Woreda, Amhara Regional State, Northwest, Ethiopia, 2014. Journal of Gynecology and Obstetrics. Vol. 3, No. 2, 2015, pp. 21-25. doi: $10.11648 /$ j.jgo.20150302.11 